\title{
Adjacent Stimulation and Measurement Patterns Considered Harmful
}

\author{
Andy Adler ${ }^{1}$, Pascal O. Gaggero ${ }^{2}$, Yasheng Maimaitijiang ${ }^{1}$ \\ ${ }^{1}$ Systems and Computer Engineering, Carleton University, Ottawa, Canada ${ }^{2}$ Centre \\ suisse d'electronique et de microtechnique (CSEM), Landquart, Switzerland \\ E-mail: $\quad$ adler, mamatjan\}@sce.carleton.ca, pascal.gaggero@csem.ch
}

\begin{abstract}
We characterize the ability of electrical impedance tomography (EIT) to distinguish changes in internal conductivity distributions, and analyze it as a function of stimulation and measurement patterns. A distinguishability measure, $z$, is proposed which is related to the signal to noise ratio of a medium and to the probability of detection of conductivity changes in a region of interest. $z$ is a function of the number of electrodes, the EIT stimulation and measurement protocol, the stimulation amplitude, the measurement noise, and the size and location of the contrasts. Using this measure we analyze various choices of stimulation and measurement patterns under the constraint of medical electrical safety limits (maximum current into the body). Analysis is performed for a planar placement of 16 electrodes for simulated 3D tank and chest shapes, and measurements in a saline tank. Results show that the traditional (and still most common) adjacent stimulation and measurement patterns have by far the poorest performance (by 6.9×). Good results are obtained for trigonometric patterns and for pair drive and measurement patterns separated by over $90^{\circ}$. Since the possible improvement over adjacent patterns is so large, we present this result as a call to action: adjacent patterns are harmful, and should be abandoned. We recommend using pair drive and measurement patterns separated by one electrode less than $180^{\circ}$. We describe an approach to modify an adjacent pattern EIT system by adjusting electrode placement.
\end{abstract}

Keywords: Electrical Impedance Tomography, distinguishability Stimulation and Measurement patterns

\section{Introduction}

Electrical Impedance Tomography (EIT) seeks to distinguish internal conductivity distributions from current stimulation and electrical measurement at electrodes on the surface. EIT has the advantage of being non-invasive, using non-ionizing radiation, with relatively low cost electronics and small size. Based on these advantages, EIT has been proposed primarily as a monitoring and assessment tool with medical applications such as detecting cancerous breast tissue or imaging the distribution of ventilation and perfusion in the thorax (Holder, 2005). Given these applications, the primary measure of the quality of an EIT system is thus its distinguishability: i.e. can it distinguish a 
pattern of clinical interest from a similar, but benign one? To this primary quality feature may be added other features such as the quality of images and the severity of reconstruction artefacts, such as position error, ringing and movement artefacts (for example as defined by Adler et al 2009).

An optimized EIT system will thus have large distinguishability, which we show is determined by the current stimulation amplitude, the accuracy of voltage measurement, the number and placement of electrodes, and the stimulation and measurement patterns. This paper considers optimization of stimulation and measurement patterns (afterward referred to as "interface" patterns). EIT images have low spatial resolution due to limited number of independent measurements, and thus the spatial resolution is thus one of the key parameters characterising an EIT system. Both resolution and distinguishability depend on the choice of interface patterns. Such considerations, and the potential trade-off between them have been identified in the early EIT literature (for example by Gisser et al 1987, and Eyüboğlu and Pilkington 1993). Two broad categories of interface patterns may be identified based on type of current drive: 1) Pair drive systems use only two electrodes at a time for current stimulation, while measurements are typically made at all electrodes not used for stimulation; and 2) more complex designs, such as for optimal pattern generation, which stimulate current on all electrodes simultaneously, and measure voltage at the driven electrodes. The design of pair drive instrumentation is considerably less complex, since current generators do not need to be matched, and the effect of contact impedance on measurements is reduced. Traditionally, since the pioneering work of Barber et al (1983) the majority of EIT systems, especially for thoracic imaging, have placed equispaced electrodes in a single plane surrounding the body, and used adjacent current stimulation and differential voltage measurement patterns (the "Sheffield protocol"). This choice was driven primarily by the requirements of the Sheffield backprojection algorithm (Barber and Seagar, 1987). However, it has continued to be used in new EIT designs, even though the regularized reconstruction approaches (developed since the work of Murai and Kagawa, 1985) are inherently agnostic of the details of the interface patterns. Of the recent EIT designs of which the authors are aware (both commercial and research), three quarters use the Sheffield protocol. In contrast, geophysical EIT systems have little used adjacent patterns (Dahlin and Zhou 2004).

Unfortunately, as we show, adjacent interface patterns are a poor design. During this research, it did not so much surprise us that adjacent patterns were suboptimal, but rather the extent of the effect. The signal to noise ratio (SNR) can be improved by a large factor by simply changing patterns. We are thus motivated to present this work as a call to action: adjacent interface patterns dramatically decrease EIT's performance; we consider them harmful.

The paper is organized as follows: first, we review formulations of distinguishability and develop a measure that may be directly evaluated on both a simulation model and in phantom systems. Next, we evaluate simulation model systems of the thorax and phantom tanks, and compare these results to measures in a phantom system. We 
interpret these results in terms of their significance for EIT interface patterns and make recommendations for EIT design. We conclude by describing a way to adapt a system based on the Sheffield protocol to increase its distinguishability.

\section{Distinguishability Measures}

Figure 1 outlines our conceptual formulation of distinguishability measurement. Two configurations of the medium are possible, $H_{0}$ and $H_{1}$, with conductivity distributions $\boldsymbol{\sigma}_{0}$ and $\boldsymbol{\sigma}_{1}$, which differ by a change in conductivity $\Delta \boldsymbol{\sigma}=\boldsymbol{\sigma}_{1}-\boldsymbol{\sigma}_{0}$ in a region of interest (ROI). Based on EIT measurements, we attempt to distinguish these configurations to test whether the configuration is $H_{0}$ or $H_{1}$. Clearly, a system with good distinguishability will make this determination accurately, while a system with poor distinguishability with perform this task with a higher probability of error. This formulation is based on previous models (Isaacson, 1986; Eyüboğlu and Pilkington, 1993; and Lionheart et al 2001)

We consider an EIT system which attaches $N_{e}$ electrodes to the boundary, $\partial \Omega$ of an object $\Omega$. During stimulation pattern $k$, a data set $\mathbf{d}^{k}$ is acquired by applying a current vector $\mathbf{c}^{k}$ to the electrodes, where each element $j,\left[\mathbf{c}^{k}\right]_{j}$, represents the phasor current amplitude into electrode $j$. By Kirchhoff's current law, $\sum\left[\mathbf{c}^{k}\right]_{j}=0$. A pair drive stimulation of current $I$ at electrodes $c_{+}, c_{-}$will thus have $\left[\mathbf{c}^{k}\right]_{c_{+}}=+I,\left[\mathbf{c}^{k}\right]_{c_{-}}=-I$ and $\left[\mathbf{c}^{k}\right]=0$ elsewhere.

Such stimulation produces a distribution of voltage $\mathbf{v}^{k}=\mathbf{T}(\boldsymbol{\sigma}) \mathbf{c}^{k}$ at the electrodes, where the voltage at electrode $j$ is $\left[\mathbf{v}^{k}\right]_{j}$, and $\mathbf{T}(\boldsymbol{\sigma})$ is the transfer impedance matrix which depends on the (possibly complex) conductivity distribution $\boldsymbol{\sigma}$ in $\Omega$. Most EIT

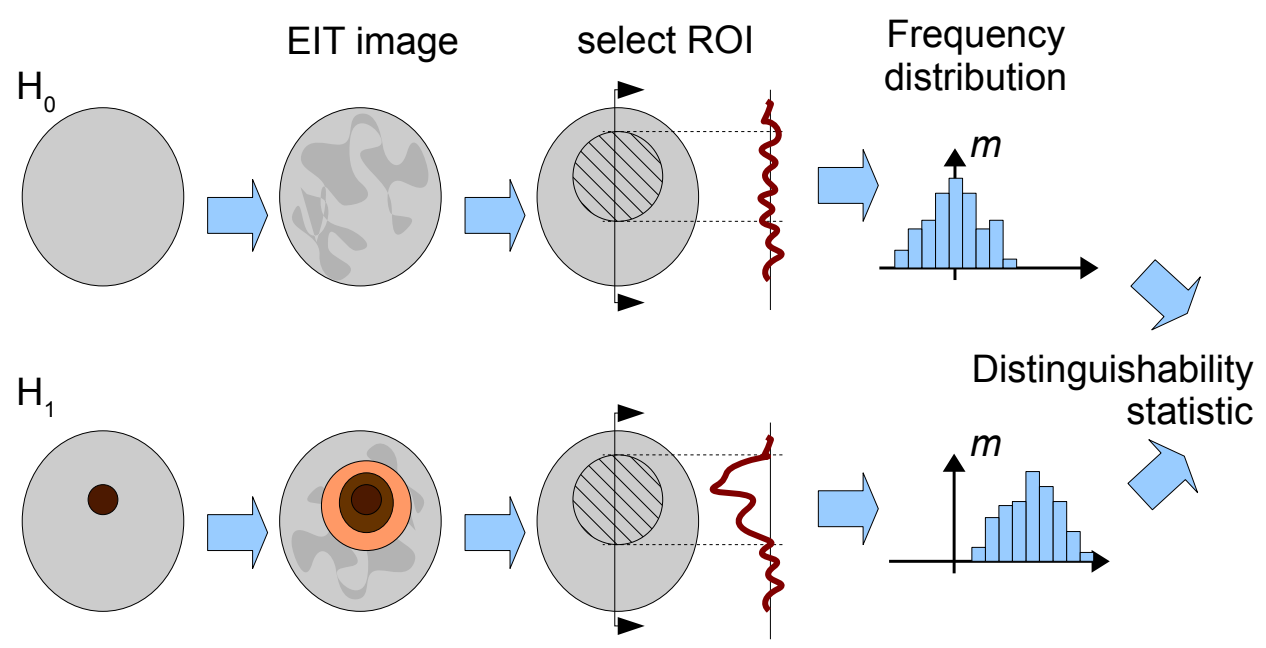

Figure 1. Conceptual diagram of distinguishability. Images $H_{0}$ and $H_{1}$ with conductivity distributions $\boldsymbol{\sigma}_{0}$ and $\boldsymbol{\sigma}_{1}$ are to be distinguished based on their EIT images. Over a large sequence of tests of each case, image are generated and the distribution of a measurement $m$ in the ROI is calculated, from which the distinguishability statistic is calculated. 
systems do not directly measure voltage, but rather use differential instrumentation amplifiers to measure differences between electrode pairs. The measured data set, $\mathbf{d}^{k}$ is related to the electrode voltages by a measurement protocol $\mathbf{M}$. If the $i^{\text {th }}$ measurement attaches the differential amplifier to electrodes $m_{+}, m_{-}$, then $[\mathbf{M}]_{i, m_{+}}=+G,[\mathbf{M}]_{i, m_{-}}=$ $-G$ and the other values on row $i$ are zero ( $G$ represents the channel gain). For systems which do not measure at stimulation electrodes, $\mathbf{M}$ is zero in columns $c_{+}, c_{-}$.

To the measured data, an EIT system adds a certain level of random noise $\mathbf{n}^{k}$, which we characterize as zero-mean and Gaussian with co-variance $\boldsymbol{\Sigma}_{k}$. This characterization is justified as follows: the noise has zero-mean because any average bias can be measured and incorporated into the EIT model. The assumption of Gaussian noise is designed to make the statistical computations easier; however, there is some evidence to indicate that EIT noise is not Gaussian (Hahn et al 2008), and specifically, large errors occur far more often than would be predicted by a Gaussian statistics. Noise samples may be correlated between measurements due to the design of amplifier and analog switch circuitry, giving non-zero off diagonal values in $\boldsymbol{\Sigma}_{k}$ :

$$
\mathbf{d}_{k}=\mathbf{M}_{k} \mathbf{T}(\mathbf{s}) \mathbf{c}_{k}+\mathbf{n}_{k} .
$$

The scenarios to distinguish, $H_{0}$ and $H_{1}$ with conductivity distributions $\boldsymbol{\sigma}_{0}$ and $\boldsymbol{\sigma}_{1}$, yield EIT measurements which differ for the same $\mathbf{c}_{k}$,

$$
\Delta \overline{\mathbf{d}}_{k}=\overline{\mathbf{d}}_{k}\left(\boldsymbol{\sigma}_{1}\right)-\overline{\mathbf{d}}_{k}\left(\boldsymbol{\sigma}_{0}\right)=\mathbf{M}_{k}\left(\mathbf{T}\left(\boldsymbol{\sigma}_{1}\right)-\mathbf{T}\left(\boldsymbol{\sigma}_{0}\right)\right) \mathbf{c}_{k}=\mathbf{T}_{\Delta} \mathbf{c}_{k}
$$

where the change in transfer impedance, $\mathbf{T}_{\Delta}=\mathbf{T}\left(\boldsymbol{\sigma}_{1}\right)-\mathbf{T}\left(\boldsymbol{\sigma}_{0}\right)$, may be determined experimentally or calculated from finite element model (FEM) or analytic techniques.

Isaacson (1989) developed the earliest formulation of distinguishability in EIT, in which $\left\|\Delta \mathbf{d}^{k}\right\|$ is maximized subject to a constraint, $l$, limiting the maximum power in the stimulation current: $\left\|\mathbf{c}^{k}\right\|_{2} \leq l$. This limit implies that the best $\mathbf{c}^{k}$ corresponds to the largest eigenvector of $\mathbf{T}_{\Delta}$, which was shown to be a spatially sinusoidal pattern for a circular 2D system with a central anomaly. This result may be motivated as follows: using the singular value decomposition (SVD), the symmetric $\mathbf{T}_{\Delta}=\mathrm{VDV}^{\mathbf{t}}$ and $\left\|\Delta \mathbf{d}^{k}\right\|=\mathbf{c}^{t} \mathrm{VD}^{2} \mathrm{~V}^{t} \mathbf{c}$, and the optimal $\mathbf{c}$ corresponds to the largest value in $\mathbf{D}$.

Lionheart et al (2001) extended this formulation to consider various safety constraints. Three optimal criteria are considered, which limit (i) the maximum ohmic power dissipated in the body $\left(\left\|\mathbf{c}^{k}\right\|_{2} \leq l\right.$ ), (ii) the maximum total current into the body $\left(\left\|\mathbf{c}^{k}\right\|_{1} \leq l\right)$, and (iii) maximum current for each drive channel $\left(\left\|\mathbf{c}^{k}\right\|_{\infty} \leq l\right)$. Based on current international electrical safety standards (IEC 60601-1, 2005) the limits to currents used in EIT are based on the patient auxiliary current, defined as

current flowing in the patient in normal use between any patient connection and all other patient connections and not intended to produce a physiological effect.

We interpret this to correspond most closely to case (ii) which considers the sum of applied current, since current is measured "between a single part of the Applied Part and all other Applied Parts connected together". However, we acknowledge that 
this standard could also be interpreted as a requirement that the current through any particular electrode must be within the limit (case iii). In case (ii), the optimal current pattern is a pair drive. This limit may be seen by observing that, for pair drive at $\left(c_{+}, c_{-}\right),\left\|\Delta \mathbf{d}^{k}\right\|=\left[\mathbf{T}_{\Delta}\right]_{c_{+}, c_{+}}+\left[\mathbf{T}_{\Delta}\right]_{c_{-}, c_{-}}-2\left[\mathbf{T}_{\Delta}\right]_{c_{+}, c_{-}}$. The $\left(c_{+}, c_{-}\right)$which maximize this value can be found by exhaustive search. If current at these electrodes is then reduced to apply current elsewhere, the contribution to $\left\|\Delta \mathbf{d}_{k}\right\|$ cannot increase since the newly stimulated electrodes cannot provide a larger contribution than the maximal location. If IEC 60601 is interpreted to limit the current in each electrode (case iii), then optimal patterns will apply \pm the maximum current to each electrode.

These analyses of distinguishability have two limitations which we address in the next section. They consider only a single current stimulation pattern rather than data from a complete set of EIT stimulation patterns; and distinguishability criteria are formulated at the level of the raw data rather than the EIT images.

\section{Distinguishability}

From the EIT images in figure 1, we wish to distinguish $H_{1}$ from $H_{0}$ using a measurement $m$ of the average EIT image in the ROI. The null hypothesis $H_{0}$ may be rejected with a probability given by the $z$-score

$$
z=\frac{\Delta \bar{m}}{\sigma_{m}}
$$

where $\Delta \bar{m}=\bar{m}_{1}-\bar{m}_{2}$ is the difference between mean $m$ in the scenarios and $\sigma_{m}$ is the standard deviation. The use of normal statistics is appropriate, as the noise for a given EIT system can be well characterized and will be equal under both scenarios. As $z$ increases, the probability of error decreases corresponding to the increased capability of EIT distinguish. Note that $z$ may also be interpreted as SNR.

An EIT system measures a set or frame of data, d, by applying, in sequence, $N_{c}$ current patterns and measuring the output, yielding a column concatenated data set $\mathbf{d}=\left[\mathbf{d}_{1}^{t}\left|\mathbf{d}_{2}^{t}\right| \cdots \mid \mathbf{d}_{N_{c}}^{t}\right]^{t}$. This EIT system is characterized by $F(\cdot)$ where

$$
\mathbf{d}=F(\boldsymbol{\sigma})+\mathbf{n}
$$

where noise, $\mathbf{n}$ has zero mean and a block diagonal covariance $\boldsymbol{\Sigma}$ with blocks $\boldsymbol{\Sigma}_{k}, 1 \leq$ $k \leq N_{c}$.

We assume that the distinguishability problem consists of a small $\Delta \boldsymbol{\sigma}$ for which a linearized reconstruction is valid. Difference measurements $\Delta \mathbf{d}=\mathbf{d}-\mathbf{d}_{0}$ are made between the current (unknown) distribution and a reference distribution known to match $H_{0}$. We further assume that data $\mathbf{d}_{0}$ represent the average of an extended calibration measurement, so that the noise contribution is exclusively due to $\mathbf{d}$. Linearizing around $\boldsymbol{\sigma}_{0}$, we express

$$
\Delta \mathbf{d}=\mathbf{J} \Delta \boldsymbol{\sigma}+\mathbf{n},
$$


where $\mathbf{J}$ is the Jacobian (sensitivity) defined as

$$
[\mathbf{J}]_{i, j}=\left.\frac{\partial F_{i}(\boldsymbol{\sigma})}{\partial \boldsymbol{\sigma}_{j}}\right|_{\boldsymbol{\sigma}=\boldsymbol{\sigma}_{0}}
$$

This linearization is appropriate because the distinguishability limits for most EIT systems, correspond to relatively small changes in conductivity.

From measurements $\Delta \mathbf{d}$, an impedance change image estimate $\Delta \hat{\boldsymbol{\sigma}}$ is reconstructed from a linearized difference EIT reconstruction algorithm as $\Delta \hat{\boldsymbol{\sigma}}=\mathbf{R} \Delta \mathbf{d}$, defined from

$$
\Delta \hat{\boldsymbol{\sigma}}=\underset{\Delta \boldsymbol{\sigma}}{\arg \min }\|\Delta \mathbf{d}-\mathbf{J} \Delta \boldsymbol{\sigma}\|_{\boldsymbol{\Sigma}^{-1}}+P(\mathbf{x})
$$

where $\arg \min _{\Delta \boldsymbol{\sigma}}$ selects the value of the parameter $(\Delta \boldsymbol{\sigma})$ which minimizes the expression which follows, and $P(\cdot)$ represents a penalty or regularization term. For quadratic $P(\cdot)$, image reconstruction may be represented as multiplication by a reconstruction matrix. Such linear reconstruction matrix can also describe the majority of difference EIT reconstruction algorithms, such as the Sheffield Backprojection (Barber and Seagar, 1987) and many regularization based schemes.

In general, we are interested in investigating the image output within a ROI of area $A_{R}$, defined by a vector $\boldsymbol{\Theta}_{R}$, of size $N \times 1$ in which each element $\left[\mathbf{I}_{R}\right]_{i}$ is the area fraction of element in the ROI, and is zero outside the ROI. Based on the ROI, we define the measurement $m$ as the area weighted average impedance change in the ROI $\left(\Delta \bar{\sigma}_{R}\right)$ :

$$
m=\boldsymbol{\Theta}_{R}^{t} \Delta \hat{\boldsymbol{\sigma}}=\boldsymbol{\Theta}_{R}^{t} \mathbf{R} \Delta \mathbf{d}=\mathbf{R}_{R} \Delta \mathbf{d}=A_{R} \Delta \bar{\sigma}_{R}
$$

where $\mathbf{R}_{R}=\boldsymbol{\Theta}_{R}^{t} \mathbf{R}$ is the reconstruction matrix of the scalar measurement $m$; this scalar reconstruction makes the problem not ill-posed and no regularization term $P(\cdot)$ is required, and the maximum likelihood solution matrix, $\mathbf{R}_{R}$, is given by:

$$
\mathbf{R}_{R}=\left(\mathbf{J}_{R}^{t} \boldsymbol{\Sigma}^{-1} \mathbf{J}_{R}\right)^{-1} \mathbf{J}_{R}^{t} \Sigma^{-1}
$$

where $\mathbf{J}_{R}=\frac{1}{A_{R}} \mathbf{J} \Theta_{R}$, since we require $\Delta \mathbf{d}=\mathbf{J}_{R} m$ for changes in the ROI.

\subsection{Formulation of Distinguishability}

Using this formulation, the values in (3) are calculated. In order to distinguish $\boldsymbol{\sigma}_{1}$ from

$\boldsymbol{\sigma}_{0}$, we must reject the null hypothesis $H_{0}: m=0$. Thus $\Delta m=m$, and the probability of $H_{0}$ is based on the $z$-score: $z=\bar{m} / \sigma_{m}$, where

$$
\bar{m}=E[m]=E\left[\mathbf{R}_{R}(\Delta \mathbf{d}+\mathbf{n})\right]=\mathbf{R}_{R} \Delta \mathbf{d}=\mathbf{R}_{R} \mathbf{J} \Delta \bar{\sigma}_{R}=A_{R} \Delta \bar{\sigma}_{R}
$$

and

$$
\begin{aligned}
\operatorname{var}(m) & =\sigma_{m}^{2}=E\left[\|m-\bar{m}\|^{2}\right]=E\left[\left\|\mathbf{R}_{R} \mathbf{n}\right\|^{2}\right]=E\left[\mathbf{R}_{R} \mathbf{n n}^{t} \mathbf{R}_{R}^{t}\right] \\
& =\mathbf{R}_{R} \boldsymbol{\Sigma}_{n} \mathbf{R}_{R}^{t}
\end{aligned}
$$

where $E[\cdot]$ is the expected value.

Given the maximum likelihood solution for $m$, we further simplify $\sigma_{m}^{2}$ as

$$
\begin{aligned}
\sigma_{m}^{2} & =\mathbf{R}_{R} \boldsymbol{\Sigma}_{n} \mathbf{R}_{R}^{t}=\left(\mathbf{J}_{R}^{t} \boldsymbol{\Sigma}_{n}^{-1} \mathbf{J}_{R}\right)^{-1} \mathbf{J}_{R}^{t} \boldsymbol{\Sigma}_{n}^{-1} \boldsymbol{\Sigma}_{n} \boldsymbol{\Sigma}_{n}^{-1} \mathbf{J}_{R}\left(\mathbf{J}_{R}^{t} \boldsymbol{\Sigma}_{n}^{-1} \mathbf{J}_{R}\right)^{-1} \\
& =\left(\mathbf{J}_{R}^{t} \boldsymbol{\Sigma}_{n}^{-1} \mathbf{J}_{R}\right)^{-1}
\end{aligned}
$$


The $z$ score may be calculated as

$$
\begin{aligned}
z & =\frac{\bar{m}}{\sigma_{m}}=\frac{A_{R} \Delta \bar{\sigma}_{R}}{\left(\mathbf{R}_{R}^{t} \boldsymbol{\Sigma}_{n} \mathbf{R}_{R}\right)^{\frac{1}{2}}}=A_{R} \Delta \bar{\sigma}_{R} \sqrt{\mathbf{J}_{R}^{t} \Sigma_{n}^{-1} \mathbf{J}_{R}}=\sqrt{\Delta \mathbf{d} \Sigma_{n}^{-1} \Delta \mathbf{d}} \\
& =\|\Delta \mathbf{d}\|_{\boldsymbol{\Sigma}_{n}^{-1}}
\end{aligned}
$$

since $\Delta \mathbf{d}=\mathbf{J}_{R} A_{R} \Delta \bar{\sigma}_{R}$.

This result shows that the distinguishability is a product of the target size and amplitude $(m)$, the measurement strategy $(\mathbf{J})$ and the inverse of the noise amplitude $(\boldsymbol{\Sigma})$. Compared to the signal $\|\mathbf{v}\|$ defined by Isaacson (1986), the definition (13) reflects the measurement scheme, the noise properties, and the information from multiple current patterns.

\subsection{Distinguishability of Current Patterns}

This formulation may be extended to clarify the effect of the choice of stimulation and measurement patterns on the SNR and z. Equation (13) may be separated into the components from each stimulation pattern $k$.

$$
z^{2}=\|\Delta \mathbf{d}\|_{\Sigma_{n}^{-1}}^{2}=\sum_{k}\left\|\Delta \mathbf{d}_{k}\right\|_{\Sigma_{k}^{-1}}^{2}=\sum_{k}\left\|\mathbf{M}_{k} \mathbf{T}_{\Delta} \mathbf{c}\right\|_{\Sigma_{k}^{-1}}^{2}
$$

As mentioned, most EIT systems have independent noise, which is often equal for all channels. Some systems vary the gain for each measurement to compensate for the large dynamic range (i.e. Sheffield Mk I, (Brown and Seagar, 1987)) or explicitly filter measurements (i.e. Hartinger, 2007). In general, a noise-normalized measurement

sequence may be calculated as $\hat{\mathbf{M}}_{k}=\mathbf{S}_{k}^{-\frac{1}{2}} \mathbf{V}_{k}^{t} \mathbf{M}_{k}$ based on the SVD, $\boldsymbol{\Sigma}_{k}=\mathbf{V}_{k} \mathbf{S}_{k} \mathbf{V}_{k}^{t}$. Thus

$$
z^{2}=\sum_{k}\left\|\hat{\mathbf{M}}_{k} \mathbf{T}_{\Delta} \mathbf{c}_{k}\right\|^{2}=\sum_{k} \sum_{j}\left(\hat{\mathbf{m}}_{k, j} \mathbf{T}_{\Delta} \mathbf{c}_{k}\right)^{2}
$$

where $\hat{\mathbf{m}}_{k, j}$ is the $j^{\text {th }}$ row of $\hat{\mathbf{M}}_{k}$, which is measurement at the $k^{\text {th }}$ of current pattern.

Equation (15) shows how measurements combine to produce the total system distinguishability. The signals with the highest SNR have the greatest impact, while noisier signals contribute little to the system performance. In most EIT systems, noise is independent and equal across channels, and $\hat{\mathbf{M}}=\sigma^{-1} \mathbf{M}$. It is also worth noting that, since $\mathbf{T}_{\Delta}$ is symmetric, measurements and current patterns play an equivalent role in calculation of distinguishability. This function may be regarded as a compliment to an analysis of the information content of EIT signals (Adler and Lionheart, 2008).

\section{Methods}

\subsection{Simulation}

In this section, we explore the behaviour of (15) for different injection and measurement patterns. In all cases, total current into the body is set to $10 \mathrm{~mA}$ as the maximum 
patient auxiliary current specified by IEC 60601-1 (2005). For bipolar stimulations, this represents $10 \mathrm{~mA}$ flowing from one stimulation electrode to the other per electrode, while for sinusoidal patterns, the total current through "all other applied parts" is $\|\mathbf{c}\|_{1}=10 \mathrm{~mA}$. Noise was assumed to be independent and Gaussian with a $100 \mu \mathrm{V}$ standard deviation. This represents a moderate performance EIT system (Gagnon et $a l, 2010)$, and the small simulated targets (2\% of medium diameter) are on the limit of detectability).

Simulations are conducted with EIDORS (Adler and Lionheart, 2006) using FE models created by Netgen (Schöbel, 1997). We consider only electrode placements of 16 equispaced electrodes in a single plane around the body, since this corresponds to the most common EIT systems for lung imaging. Two different models are simulated as shown in figure 2: 1) a uniform cylindrical tank of $50 \mathrm{~cm}$ diameter and $50 \mathrm{~cm}$ height, and 2) a model based on a computed tomography (CT) section of the thorax scaled to $50 \mathrm{~cm}$ diameter and vertically extruded to $50 \mathrm{~cm}$ height. The thorax model was based (with consent) on CT data from a healthy male subject. Lung regions were modelled to have a conductivity of $0.12 \mathrm{~S} / \mathrm{m}$, while other chest tissue was assigned a conductivity of $0.48 \mathrm{~S} / \mathrm{m}$ (based on our model of Adler et al 1996). Models were refined in the vicinity of electrodes to improve model accuracy in regions of high electric field.
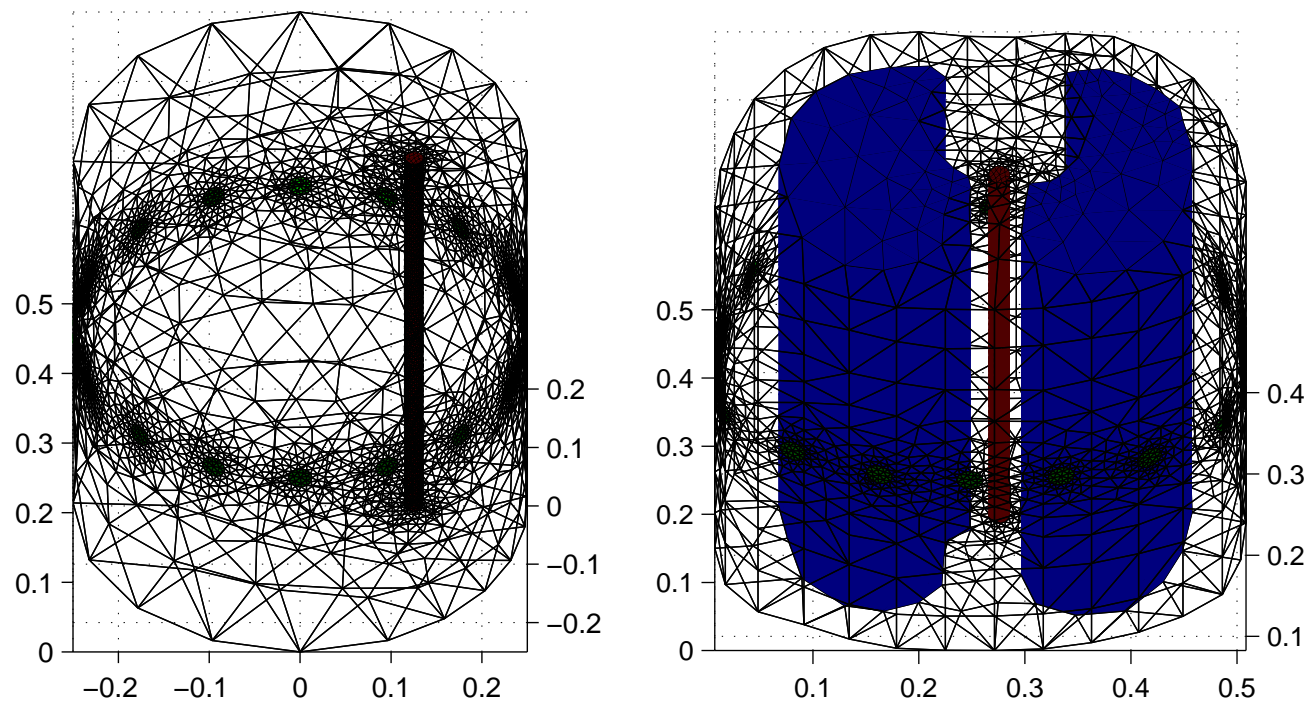

Figure 2. Finite element models used for simulations of distinguishability. In each case, a small cylindrical contrasting region (red) is shown. The contrast region is moved from the model centre to the right (patient left) electrode, and the normalized target position, $\hat{r}$ is defined as a fraction of the distance from the centre to the medium boundary. Left: uniform cylindrical FEM. Right: chest body shaped FEM with lower conductivity lung regions.

Contrasting regions were created in the FE models by definition of a small $(1.0 \mathrm{~cm}$ diameter, or $2 \%$ of medium diameter) cylindrical region with very low conductivity $(0.005 \mathrm{~S} / \mathrm{m})$. The FEM region of the conductivity contrast is placed in an axis from medium centre $(\hat{r}=0)$ to the image right (or patient left), such that $\hat{r}=1$ where 
a normalized radius $\hat{r}=r / r_{\max }$. FEM element size was refined in the target region to improve model accuracy. Transfer impedance values $\mathbf{T}_{0}$ were simulated from the model with background conductivity, while $\mathbf{T}_{1}$ values were simulated by non-conductive regions in the target cylinder. Each different image was calculated from difference data simulated with the same FEM geometry; i.e. we did not compute difference images from models with different mesh geometries to avoid noise contribution described by Adler and Lionheart (2010).
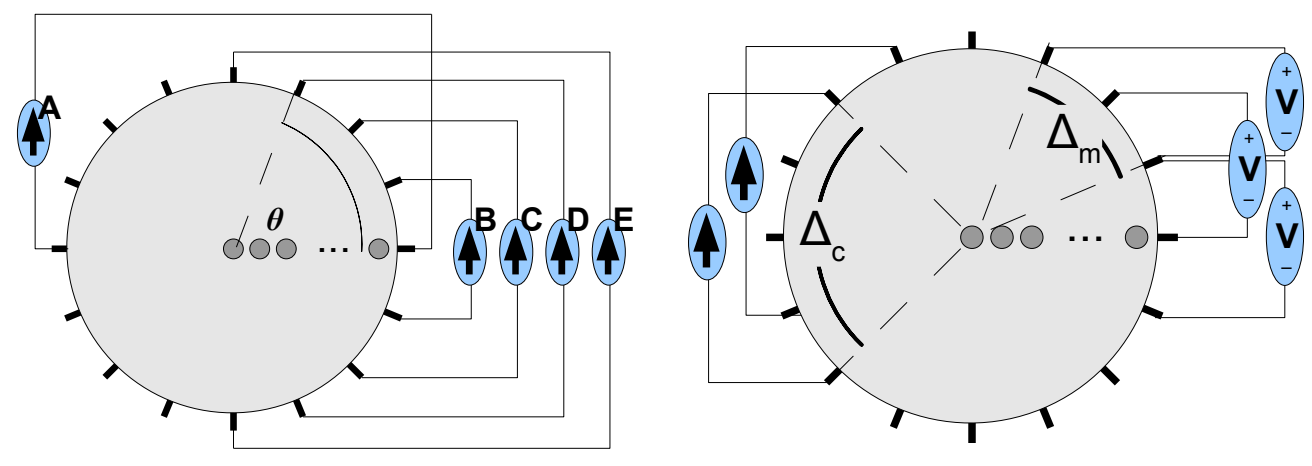

Figure 3. Block diagram of interface patterns tested. A cylindrical tank with 16 electrodes has a contrasting target moved from centre $\hat{r}=0$ to the right edge $\hat{r}=1.0$. Left: Individual stimulation patterns. Right: Bipolar interface patterns. A complete set of stimulations (with offset $\Delta_{c}$ ) and measurements (with offset $\Delta_{m}$ ) are made.

\subsection{Experimental Evaluation}

The experimental system in this study consists of a saline tank phantom, a test target and an EIT measurement system. The saline phantom with $14 \mathrm{~cm}$ radius and $36 \mathrm{~cm}$ height was equipped with 4 rows of 32 electrodes. However, only one-layer 16-electrode was used for this experiment. A non-conductive plastic cube of $50 \mathrm{ml}$ used in this test has a resistivity value of much greater than $1 M \Omega \cdot \mathrm{cm}$. The plastic target moved from center to border for $4(x, y, z)$ positions: $(0,0,0),(0,3.8,0),(0,7.6,0)$, and $(0,11.4,0)$ in the electrode plane. The normalized position values $\hat{r}$ are $0,0.27,0.54$ and 0.8 along y axis respectively. The tank was filled with 22 litres of saline solution with conductivity $0.8 S \cdot m^{-1}$. The EIT measurement system employed was a Sigma Tome II EIT system (École Polytechnique Montréal, Canada) that was used to take measurements for different current patterns. The data was analyzed offline through EIDORS and distinguishability $z$ was calculated using (3).

\section{Results}

In order to study the effect of measurements from individual stimulation patterns, we consider the patterns of figure 3 left. Measurements are made at each individual electrode (and not considering difference measurement between electrodes). Results are 
Table 1. Distinguishability values as a function of stimulation $\left(\Delta_{c}\right)$ and measurement $\left(\Delta_{m}\right)$ pattern. Values are symmetric for a swap of $\left(\Delta_{c}, \Delta_{m}\right)$. Top right values are for target object at centre, while bottom left are for target at $\hat{r}=0.75$. For comparison, trigonometric patterns yield $z=1.40(\hat{r}=0)$ and $z=5.26(\hat{r}=0.75)$.

\begin{tabular}{c|ccccccccc|c}
\hline \multicolumn{1}{c}{} & $\Delta_{c}$ & 1 & 2 & 3 & 4 & 5 & 6 & 7 & 8 & \\
\cline { 2 - 14 }$\Delta_{m}$ & & 0.28 & 0.51 & 0.79 & 1.07 & 1.33 & 1.55 & 1.69 & 1.73 & 1 \\
1 & 3.46 & 1.14 & 1.58 & 2.10 & 2.58 & 2.97 & 3.22 & 3.31 & & 2 \\
2 & 4.38 & 6.92 & 2.54 & 3.05 & 3.68 & 4.17 & 4.48 & 4.59 & & 3 \\
3 & 6.72 & 6.49 & 9.86 & 4.20 & 4.57 & 5.08 & 5.39 & 5.50 & & 4 \\
4 & 7.15 & 8.88 & 8.91 & 11.30 & 5.68 & 5.69 & 5.95 & 6.04 & & 5 \\
5 & 7.31 & 9.33 & 10.63 & 10.18 & 11.99 & 6.63 & 6.23 & 6.28 & & 6 \\
6 & 7.39 & 9.52 & 10.92 & 11.56 & 10.80 & 12.31 & 7.02 & 6.34 & & 7 \\
7 & 7.42 & 9.60 & 11.03 & 11.73 & 12.00 & 11.07 & 12.37 & 7.77 & & 8 \\
8 & 7.43 & 9.63 & 11.07 & 11.77 & 12.08 & 12.11 & 9.93 & 12.61 & & $\Delta_{m}$ \\
\cline { 2 - 11 } & 1 & 2 & 3 & 4 & 5 & 6 & 7 & 8 & $\Delta_{c}$ &
\end{tabular}

shown normalized with respect to the relevant opposite pattern $(A)$ for each electrode position. Results are shown in figure 4 for measurements (left) without and (right) with measurements on stimulated electrodes. Results for three low order trigonometric patterns are shown. Results show that stimulation patterns with lower angles yield higher $z$ only when the target is close to the boundary.
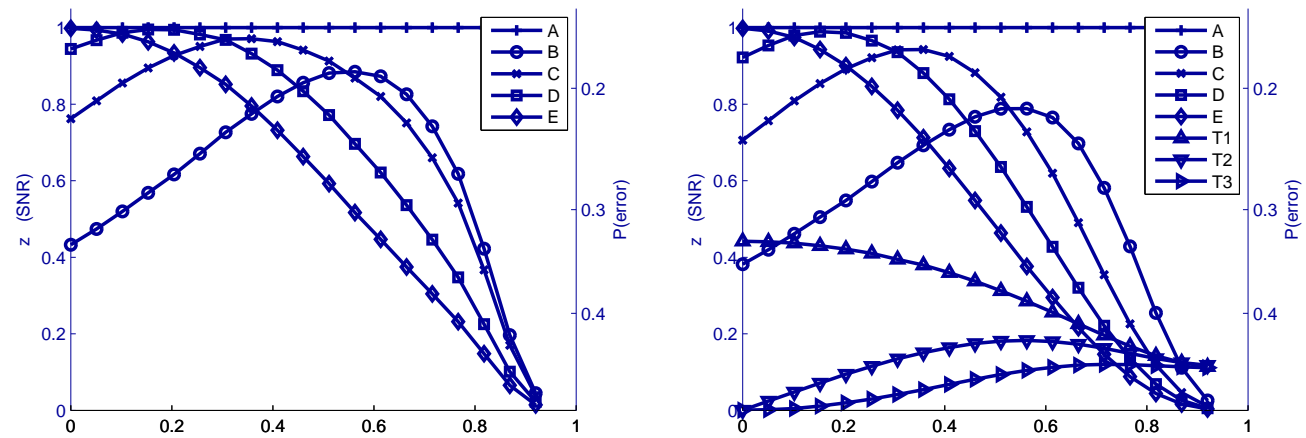

Figure 4. Normalized distinguishability $(z)$ as a function of target radial position on a cylindrical tank, for the stimulation patterns of 3 (left), and trigonometric patterns, where: $T_{1}: \cos (\theta), T_{2}: \cos (2 \theta)$ and $T_{3}: \cos (3 \theta) . z$ values are normalized to the value from pattern $A$. Left: without measurements on stimulated electrodes Right: including measurements on stimulated electrodes, .

In order to calculate the distinguishability $z$ from an EIT data frame of multiple stimulation and measurement patterns, we calculate values for the complete set of target positions $\hat{r}$, stimulation $\Delta_{c}$ and measurement patterns $\Delta_{m}$. Stimulation and measurement patterns are defined by the distance between the electrode pairs used, as shown in figure 3 right. For each calculation all stimulation and measurements with 

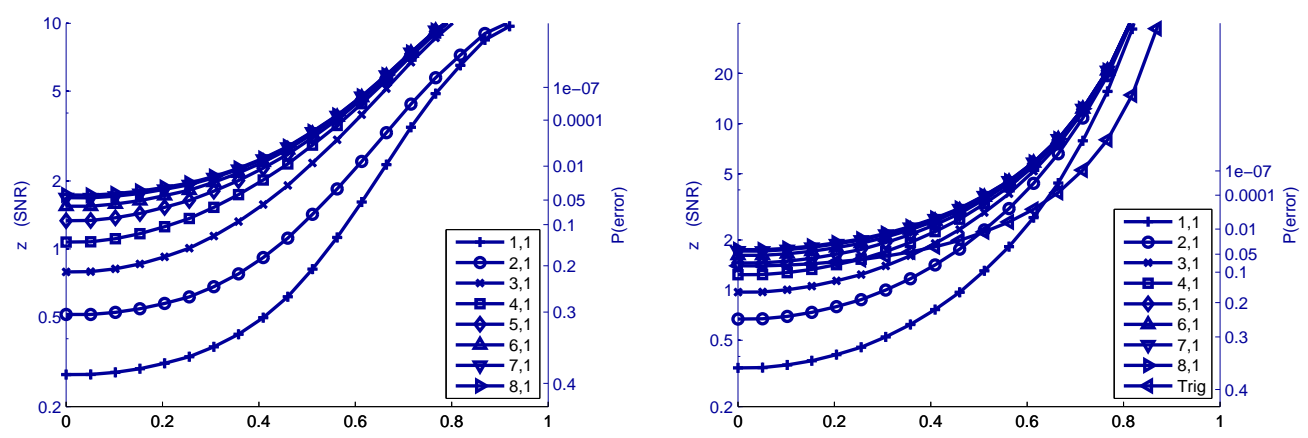

Figure 5. Distinguishability $(z)$ of a conductivity contrast of size $1.25 \mathrm{~cm}$, in a cylindrical $50 \mathrm{~cm}$ diameter tank, for the stimulation patterns of 3 (right), and the set of trigonometric patterns ("trig"). The current stimulation pattern is varied from $\Delta_{c}=1 \cdots 8$ while adjacent measurement is used $\left(\Delta_{m}=1\right)$. Left: without measurements on stimulated electrodes, Right: including measurements on stimulated electrodes.
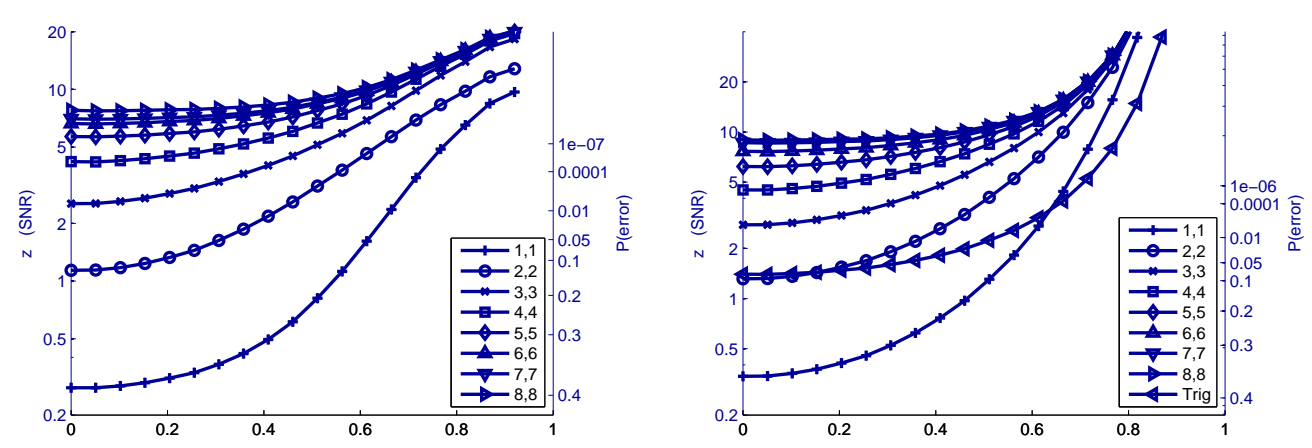

Figure 6. Distinguishability $(z)$ of a conductivity contrast of size $1.25 \mathrm{~cm}$, in a cylindrical $50 \mathrm{~cm}$ diameter tank, for the stimulation patterns of 3 (right), and the set of trigonometric patterns ("trig"). The current and measurement stimulation pattern are equal $\left(\Delta_{c}=\Delta_{m}\right)$, and are varied from $\Delta_{c}=1 \cdots 8$. Left: without measurements on stimulated electrodes, Right: including measurements on stimulated electrodes.

the given offset are calculated using (15). We show these data in several ways. Table 1 shows the values for interface patterns for $\hat{r}=0$ and $\hat{r}=0.75$. Figure 5 shows $z$ versus target position $\hat{r}$ for adjacent measurement $\left(\Delta_{m}=1\right)$ for various stimulation patterns $\left(\Delta_{s}=1 \cdots 8\right)$. Figure 6 shows $z$ versus target position $\hat{r}$ for various interface patterns $\left(\Delta_{s}=1 \cdots 8\right)$ where the stimulation and measurement patterns are equal $\left(\Delta_{s}=\Delta_{m}\right)$.

In order to explore whether such calculations differ when applied to a thorax geometry and relatively low conductivity lungs, the data of Figure 6 are recalculated for the thorax FEM figure 2 right, and shown in figure 7.

\section{Results: experimental}

Figure 8 shows the distinguishability values as a function of object positions for 8 current patterns from adjacent $\left(\Delta_{c}=1\right)$ to opposite-1 $\left(\Delta_{c}=7\right)$, using adjacent 

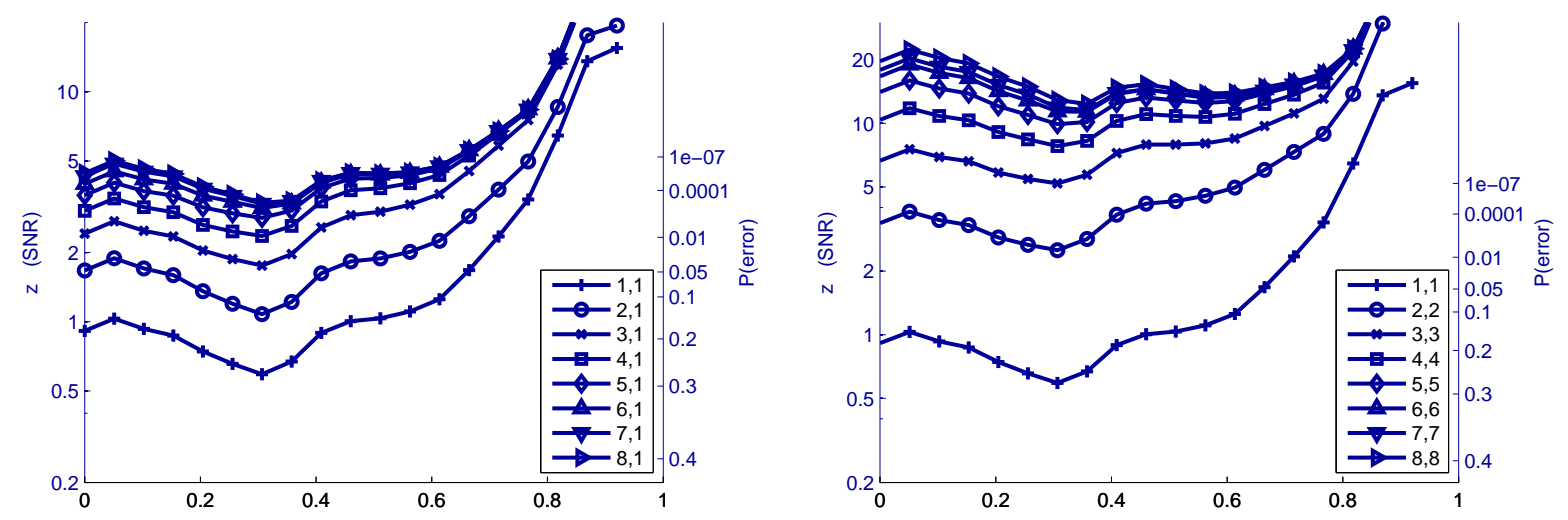

Figure 7. Distinguishability $(z)$ of a conductivity contrast of size $r=1.25 \mathrm{~cm}$ in a thorax FEM model, for the stimulation patterns of 3 (right), and the set of trigonometric patterns ("trig"). The current and measurement stimulation pattern are equal $\left(\Delta_{c}=\Delta_{m}\right)$, and are varied from $\Delta_{c}=1 \cdots 8$. Left: without measurements on stimulated electrodes, Right: including measurements on stimulated electrodes.

measurement $\left(\Delta_{m}=1\right)$. Distinguishability varied with different object positions, but higher distinguishability was obtained for the objects placed on the periphery of the tank for all current patterns. The adjacent electrode approach performed worse than all other patterns, while $\Delta_{c}=7$ performed better than all other current patterns. Error bars on figure 8 indicate the measurement error, calculated using bootstrap by random sampling with replacement from each measurement set.

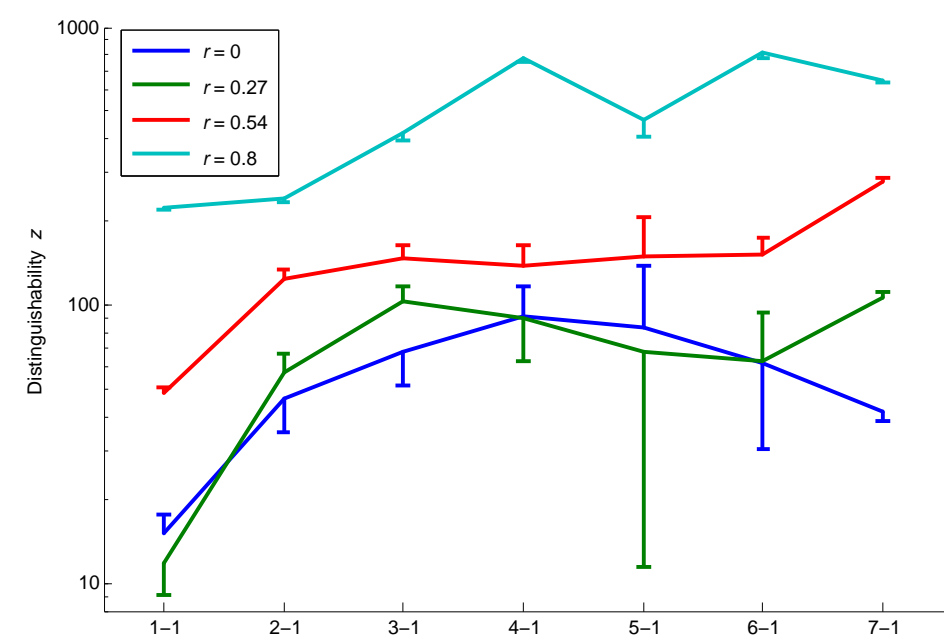

Figure 8. Distinguishability values obtained from (3), by applying current patterns $\Delta_{c}=1 \cdots 7$ and adjacent measurement. Four different target positions along $y$ coordinate of the tank and fixing its position on $\mathrm{X}$ and $\mathrm{Z}$ coordinates to 0 . Error estimates are obtained from bootstrap estimates. 


\section{Discussion}

In this paper, we aim to understand the significance of choices of the current stimulation and measurement (interface) patterns in EIT. To do this, we developed a novel approach to measurement of the distinguishability of EIT: a measure of the ability of an EIT system to detect conductivity contrasts. A measure is developed which may be calculated either experimentally, or through analysis of the transfer impedance matrix (Dirichlet to Neumann map). Using this formulation, we analyse and compare the different choices of pair drive configurations (as well as trigonometric patterns). Our approach is based on previous analysis of distinguishability (Isaacson 1986; Eyüboğlu and Pilkington 1993; and Lionheart et al 2001). The novel contribution is the formulation of the problem to include the noise covariance structure, and consideration of both measurement and stimulation patterns, which yields a result which may be interpreted as the probability of error of EIT detection. Additionally, many other papers have compared EIT stimulation patterns based on various ad-hoc criteria, such as dynamic range (i.e. Zhang and Wang, 2010).
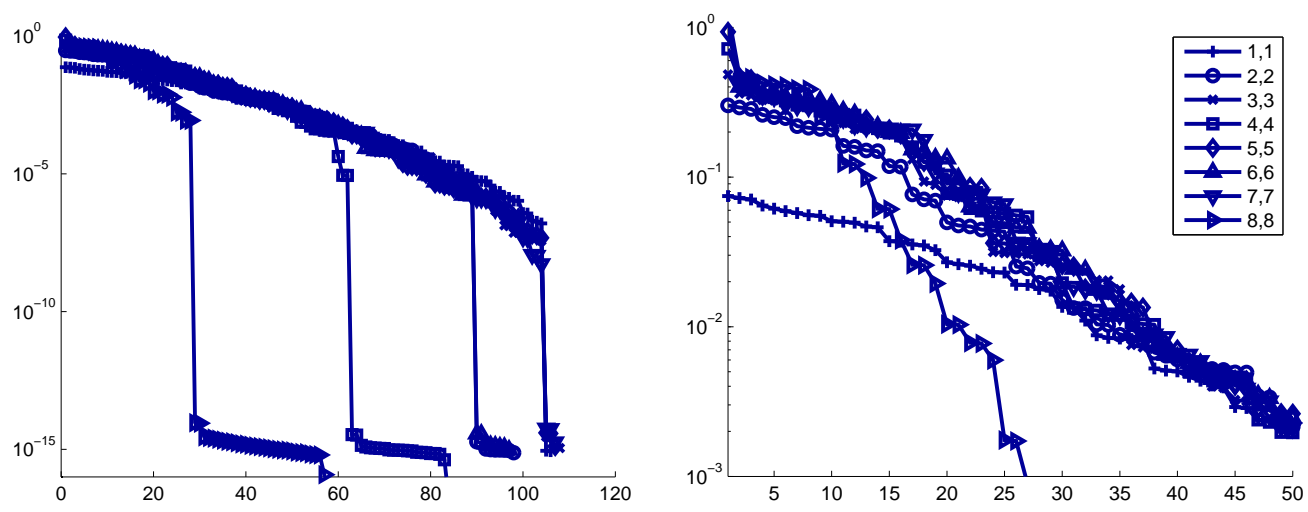

Figure 9. Amplitude of eigenvalues of the Jacobian matrix for FEM elements in the electrode plane of a cylindrical FEM. Measurement stimulation pattern are equal $\left(\Delta_{c}=\Delta_{m}\right)$, and are varied from $\Delta_{c}=1 \cdots 8$. Left: eigenvalues above the numerical resolution $\left(10^{-15}\right)$. Right: detail of eigenvalues above a more realistic noise threshold $\left(10^{-5}\right)$.

Overall, our results show that distinguishability, $z$, depends strongly on interface patterns. This effect is largest for targets in the centre, but for all target positions, the distinguishability increases as the interface pattern angle $\left(\Delta_{c}\right.$ and $\left.\Delta_{m}\right)$ increases. In general, the increase is steepest when the pattern angle is below $90^{\circ}$, after which the rate of increase is less. For thoracic shapes, the improvement of larger angle patterns over adjacent ones is approximately the same, but the spatial pattern of distinguishability varies considerably. The non-conductive lung regions have lower distinguishability than surrounding tissue. This may be explained by factors which tend to constrain current from adjacent type patterns into a narrow region near the electrodes: the noncircular shape and the presence of non-conductive lung regions. Trigonometric patterns 
outperform all adjacent measurement schemes, but have lower $z$ than patterns with $\Delta_{m}=3$. It is perhaps surprising that trigonometric patterns are not optimal under this analysis. This may be explained by noting that the first trigonometric patterns $\sin \theta, \cos$ $\theta$ are only optimal under a limitation on the maximum power applied to the body, while these results are based on the a specified maximum current into the body, based on our interpretation of IEC 60601-1 (2005). If this standard is understood to limit the current into each electrode, trigonometric patterns perform $5.0 \times$ better for a 16 electrode EIT system. Experimental evaluations agree broadly with the simulation results. In this case, $z$ values were calculated directly from analysis of mean and variance values from EIT images, as would be used to detect the presence of an unknown target contrast. Since the errors were relatively small, the calculated values of $z$ show some errors due to inaccuracies in estimates of the standard deviation.

The consequences of this work are to suggest that low angle interface patterns, and especially adjacent patterns are a poor choice. Adjacent patterns are less poor for targets close to the boundary, but this is not the case for most applications of EIT. For example, for lung imaging, conductivity changes of interest occur at $\hat{r} \leq 0.75$ for most patients. Using this estimate, we take an area average of $z$ for $\hat{r} \leq 0.75$ and calculate $z$ values for interface patterns (in parentheses): 1.09 (1), 2.90 (2), 4.57 (3), 5.77 (4), $6.58(5), 7.08(6), 7.21$ (7), and 7.56 (8); the value for trigonometric patterns is $z=5.20$. Here, it is possible to improve the performance of an adjacent patterns EIT system by $6.9 \times$ simply by changing interface strategy. Since most lung EIT systems still use such adjacent patterns, the clinical and experimental performance of EIT is thereby dramatically reduced. We take such a severe result as a call to action: we consider adjacent patterns to be harmful!

Of course, EIT is not only a technology to distinguish changes, but also to create images of the conductivity distribution. We would thus like to know how different interface patterns affect the image resolution of EIT. One way to consider the resolving capability of EIT is to look at the number of eigenvalues of the Jacobian matrix which are above the noise level. Here, using the SVD, we write $\mathbf{J}=\mathbf{U D V}^{t}$, where $\mathbf{V}$ represents decomposition of EIT image space into "eigen-EIT-images". Each eigenimage is converted into a signal via a column in $\mathbf{U}$ through an amplification in the eigenvector in the diagonal element of $\mathbf{D}$. Figure 9 shows eigenvalues for different interface patterns. One clear effect is that interface patterns which share a common factor with the number of electrodes, $N_{e}$, have far less independent patterns. This effect is most severe for opposite drive and measurement which has approximately $\frac{1}{4}$ the independent measures. In this case, EIT images will create a mirrored duplicate of target positions for such patterns. The achievable EIT resolution is limited by the noise level, since eigenvectors less than the noise level cannot be measured. Another limitation of this study is that we consider only planar electrode layouts. This reflects the most common use case for lung imaging; however, it is clearly better to use 3D electrode placement if possible.

Based on our analysis, EIT systems should avoid adjacent interface patterns. We 
recommend that interface patterns should use angles $>90^{\circ}$, but should avoid opposite and other patterns where the electrode number and pattern have common factors. Fortunately, for existing adjacent pattern EIT systems, it is normally relatively easy to follow this recommendation: electrodes can be placed at new positions, such that electrode $i$ is at position remainder $\left(N \times i, N_{e}\right)$ for values $N=7$ (for one less than opposite) or $N=5$ (for one greater than $90^{\circ}$ ).

\section{Acknowledgement}

This work was funded by a grant from NSERC Canada.

\section{References}

Adler A, Lionheart WR, Uses and abuses of EIDORS: An extensible software base for EIT, Physiol. Meas. 27:S25-S42, 2006.

Adler A, Youmaran R, Lionheart WRB, A measure of the information content of EIT data Physiol Meas, 29:S101-S109, 2008.

Adler A, et al 2009 GREIT: a unified approach to 2D linear EIT reconstruction of lung images Physiol. Meas. 30 S35-S55 169 791-800.

Adler A, Lionheart WR, Correcting for variability in mesh geometry in finite element models, 2010 J. Phys.: Conf. Ser. 224012021.

Barber D C, Brown B H and Freeston I L 1983 Imaging spatial distributions of resistivity using applied potential tomography, Electron Lett., 19, pp. 933-935.

Barber D C and Seagar A D 1987 Fast reconstruction of resistance images, Clin. Phys. Physiol. Meas. 847.

Brown B H and Seagar A D 1987 The Sheffield data collection system, Clin. Phys. Physiol. Meas. 8, Suppl. A, 91-97.

Cheney $\mathrm{M}$ and Isaacson D 1992 Distinguishability in impedance imaging IEEE-BME 39 852-860.

Dahlin T and Zhou B 2004 A numerical comparison of 2D resistivity imaging with 10 electrode arrays Geophysical Prospecting 52 379-398

Eyüboğlu BM and Pilkington T C 1993 Comment on distinguishability in electricalimpedance imaging IEEE Trans. Biomed. Eng. 40 1328-1330.

Gisser DG, Isaacson D and Newell JC 1987 Current topics in impedance imaging, Clin. Phys. Physiol. Meas., 8 Suppl A, 39-46, 1987.

Hahn G, Just A, Dittmar J, Hellige G 2008 Systematic errors of EIT systems determined by easily-scalable resistive phantoms Physiol. Meas. 29 S163-72.

Hartinger AE, Gagnon H, Guardo R 2007 Accounting for Hardware Imperfections in EIT Image Reconstruction Algorithms Physiol. Meas. 28(7), p. S13-S27.

Holder DS, (2005) Electrical Impedance Tomography : Methods, history and applications, Institute of Physics Press : London.

IEC 60601-1:2005, "Medical Electrical Equipment Part 1: General Requirements for 
Basic Safety and Essential Performance", Brussels: International Electrotechnical Commission, 2005.

Isaacson D 1986, Distinguishability of conductivities by electric current computed tomography IEEE T Med Imaging 5:91-95.

Lionheart WR, Kaipio J, McLeod CN 2001, Generalized optimal current patterns and electrical safety in EIT Physiol. Meas. 22 85-90.

Murai T and Kagawa Y 1985 Electrical impedance computed tomography based on a finite element model, IEEE Trans. Biomed. Eng. BME 32 177-84.

Schöberl J, NETGEN - An advancing front 2D/3D-mesh generator based on abstract rulesm, Computing and Visualization in Science, 1 41-52, 1997.

Yorkey T J, Webster J G and Tompkins W J 1987 Comparing reconstruction algorithms for electrical impedance tomography,IEEE Trans. Biomed. Eng 34 $843-52$.

Zhang L, Wang H, Single source current drive patterns for electrical impedance tomography Conf. Instrumentation and Measurement Technology, pp. 1477-80, Austin, USA, 2010. 